\section{Moving inhomogeneous envelopes of stars}

\author{
Lidia M. Oskinova \\ Institute for Physics and Astronomy, University of \\ Potsdam, 14476 Potsdam, Germany \\ Brankica Kubátová \\ Astronomický ústav AV ČR, Fričova 298, 25165 \\ Ondřejov, Czech Republic \\ Matematički institut SANU, Kneza Mikhaila 36, \\ 11001 Beograd, Serbia \\ Wolf-Rainer Hamann \\ Institute for Physics and Astronomy, University of \\ Potsdam, 14476 Potsdam, Germany
}

model calculations, ultimately dictate a cardinal change of this paradigm: stellar winds are highly inhomogeneous. Hence, we are now advancing to a new stage in our understanding of stellar winds. Using the foundations laid by V.V. Sobolev and his school, we now update and further develop the stellar spectral analysis techniques. New sophisticated 3-D models of radiation transfer in inhomogeneous expanding media elucidate the physics of stellar winds and improve classical empiric mass-loss rate diagnostics. Applications of these new techniques to multiwavelength observations of massive stars yield consistent and robust stellar wind parameters.

\section{Introduction}

The initial mass of a star on the zeroage main sequence largely determines its fate. Stars born with masses exceeding $\sim 10 M_{\odot}$ end their lives in a core-collapse event, e.g. a supernova (SN) explosion, and leave a neutron star or a black hole as remnant (Heger et al., 2003). Such massive stars are luminous, with bolometric luminosities exceeding $L_{\text {bol }} \gtrsim 10^{4} L_{\odot}$. On the main sequence, massive stars have spectral types earlier than B2V. These bright stars live very fast, the most massive of them die within just $\sim 10 \mathrm{Myr}$. Albeit we see many massive stars by naked eye in the night sky (e.g. the Orion Belt consists of massive stars), these stars are actually very rare and constitute only $\sim 0.4 \%$ of all stars in our Milky Way.

Despite their small number, massive stars have enormous impact on the galactic ecol- 
ogy. Their strong ionizing radiation and stellar winds, as well as their final demise in SN explosions, largely determine the physical conditions in the interstellar medium (ISM) and influence the formation of new generations of stars and planets. Thus, massive stars are among the key players in the cosmic evolution.

The atmospheres of hot massive stars are usually transparent in the continuum but opaque in many spectral lines. Because the stars are hot, a large fraction of their bolometric luminosity is emitted at ultraviolet (UV) wavelengths. The radiation leaves the star in radial direction. A photon in a spectral line $\nu_{0}$ may be absorbed by an ion and re-emitted in any direction, transferring its momentum to the ion. The ion would accelerated. Because of the Doppler effect, the wavelength of the spectral line will shift, and will be able to scatter light with wavelengths other than $\nu_{0}$. Hence, the photons within a broad wavelength range will be "swept up", by the same spectral line. The Coulomb coupling between particles ensures the collective motion, and a stellar wind develops. Such radiatively driven stellar winds (Castor et al., 1975, CAK) are ubiquitous in hot nondegenerate stars.

The amount of mass removed from the star by its wind is determined by the massloss rate, $\dot{M}$. Theory predicts that for Ostars the mass-loss rates are in the range $\dot{M}_{\mathrm{CAK}} \approx\left(10^{-7}-10^{-5}\right) M_{\odot} \mathrm{yr}^{-1}$ depending on the fundamental stellar parameters $T_{\text {eff }}, L_{\mathrm{bol}}$, and $\log g$ (Pauldrach et al., 1986; Vink et al., 2001). Hence, during stellar life time, a significant fraction of mass is removed by the stellar wind. Thus, the mass-loss rate is a crucial factor of stellar evolution.

\section{Empirical diagnostics of mass-loss}

To check and validate theoretical predictions, robust empirical estimators of massloss rates shall be employed. These diagnostics usually rely on a spectroscopic analysis. Below we briefly consider some common examples of such analyses.

\subsection{Resonance lines}

For hot stars, the resonance lines of most important ions are located in the UV part of the electromagnetic spectrum. When formed in a wind, these lines show P Cygni-type profiles (see e.g. Lamers and Cassinelli, 1999).

The resonance line of an ion is produced by photon scattering, therefore the line strength is a linear function of the density, which is related to the the expansion velocity $v(r)$ by the continuity equation

$$
\rho(r)=\frac{\dot{M}}{4 \pi v(r) r^{2}} .
$$

The radial dependence of the wind velocity is usually prescribed by the " $\beta$-velocity law", $v(r)=v_{\infty}(1-1 / r)^{\beta}$.

The line strength, the terminal wind velocity, $v_{\infty}$, and the parameter $\beta$ can be measured from the observed spectral line. Hence, in principle, by modeling a resonance line of an ion, the product of its ionization fraction and mass-loss rate could be empirically obtained. To model a spectral line, an adequate theory of line formation is required. 
Line formation in a moving stellar envelope was studied by V. V. Sobolev (Sobolev, 1947). It was shown that if the thermal motions in the atmosphere can be neglected compared to the macroscopic velocity, the radiative transfer problem can be significantly simplified (see review by Grinin, 2001). This is now known as the Sobolev approximation.

The Sobolev approximation is well justified in stellar winds, and was extensively used for their analysis. At least two different solution techniques that relied on the Sobolev approximation were developed (Castor, 1970; Lucy, 1971). An atlas of theoretical P Cygni profiles was computed (Castor and Lamers, 1979) and used to estimate mass-loss rates from the first available UV spectra of O stars (e.g. Conti and Garmany, 1980).

With time the limitations of the Sobolev approximation became clear. For instance, within a Doppler-shifts of a few tens of kilometers per second around the line center, the profiles computed in Sobolev approximation are inaccurate, especially because of the high turbulence present in stellar winds (Hamann, 1981) and/or non-monotonic wind velocities (Lucy, 1982, 1983).

These shortcomings were overcome by Hamann (1981), who compared line profiles computed with a comoving frame approach (Mihalas et al., 1975; Hamann, 1980) with those computed using Sobolev approximation. It was shown that the error in the Sobolev approximation arises mainly from the treatment of the formal integral and, to a lesser extent, from the approximated source function. Based on this suggestions Lamers et al. (1987) developed the "Sobolev with Exact Integration" method (SEI). In this method, the source function is calculated in the Sobolev approximation, but the equation of transfer is integrated exactly. As a result, the model provides significantly better fits to the observed lines (Groenewegen and Lamers, 1989), consequently allowing for more precise mass-loss rate determinations (Lamers et al., 1999).

While UV resonance lines provide excellent mass-loss rate diagnostic, there are also serious difficulties. First of all, to measure UV spectra one needs a space-based observatory. Presently, UV spectroscopy is offered only by the Hubble Space Telescope, a highly oversubscribed instrument. An even more serious problem is that in Galactic O type stars the resonance lines of the $\mathrm{CNO}$ elements are usually saturated. Therefore these lines are not sensitive to the precise values of the massloss rate and, hence, not suitable for massloss determinations.

\subsection{Stellar atmosphere model PoWR}

As an alternative to the SEI method, detailed non-LTE stellar atmosphere models for expanding atmospheres were developed in the last decades (see review by Puls et al., 2008). Such models do not rely on the Sobolev approximation but solve numerically the radiative transfer in the co-moving frame. The coupling between radiation field and statistical equations is included, leading to a highdimensional set of non-linear equations fully coupled in space and frequency. The synthetic emergent spectrum is calculated over a broad energy range. Comparing model and 
observed spectra allows to estimate stellar as well as wind parameters, specifically $\dot{M}$.

An example of such advanced stellar atmosphere model is the PoWR code (e.g. Gräfener et al., 2002; Hamann and Gräfener, 2003; Sander et al., 2015). It solves the non-LTE radiative transfer in a spherically expanding atmosphere simultaneously with the statistical equilibrium equations and accounts at the same time for energy conservation. Complex model atoms with hundreds of levels and thousands of transitions are taken into account. The extensive inclusion of the iron group elements is important not only because of their blanketing effect on the atmospheric structure, but also because the diagnostic wind lines in the UV (e.g. the CIV and Si IV resonance lines) are heavily blended with the "iron forest". X-ray emission and its effects on the ionization structure of the wind are also included in the model.

PoWR models are extensively used for the spectral analysis of stars with strong winds, such as Wolf-Rayet (WR) stars across the broad range of metallicities (Sander et al., 2012; Hainich et al., 2014, 2015). Because the hydrostatic and wind parts of the atmosphere are solved consistently (Sander et al., 2015), the PoWR models are well suited for modeling O star spectra (Evans et al., 2011; Shenar et al., 2015), as well as B star spectra (Oskinova et al., 2011b). Overall, PoWR models can be applied for spectroscopic analysis of any type of hot stars (e.g. Todt et al., 2010; Jeffery and Hamann, 2010).

\subsection{Optically thin recombination lines: $H \alpha$}

While the UV observations providing access to the resonance lines are scarce, the optical spectra of bright massive stars are easy to obtain. In $\mathrm{OB}$ supergiants, the $\mathrm{H} \alpha$ line is typically in emission. This line provides a convenient diagnostic of the wind.

In most $\mathrm{O}$ stars, the $\mathrm{H} \alpha$ line is optically thin. In this case, the line luminosity is the volume integral over the line emissivity, $j_{1}$,

$$
L_{1}=\int_{V} j_{1} \mathrm{~d} V
$$

The $\mathrm{H} \alpha$ line is mainly fed via the recombination cascade, i.e. an interaction between an electron and an ion. This is a two-body process that depends on the square of the density and is some function of the temperature, $j_{1} \sim\left\langle\rho^{2}\right\rangle f(T)$, where $\left\langle\rho^{2}\right\rangle$ is the average over the volume.

A medium with density fluctuations can be described using the wind inhomogeneity parameter (Allen, 1973)

$$
\mathcal{X} \equiv \frac{\left\langle\rho^{2}\right\rangle}{\langle\rho\rangle^{2}} .
$$

Using the continuity equation Eq. (1) for the averaged density $\langle\rho\rangle$, and combining Eqs. $(2,3)$, the mass-loss rate can be expressed as

$$
\dot{M} \propto v_{\infty} \sqrt{\frac{L_{1}}{\mathcal{X}}}
$$

where $v_{\infty}$ and $L_{1}$ can be measured from observed spectra, but the parameter $\mathcal{X}$ is largely unknown.

The value of $\mathcal{X}$ is very difficult to estimate; moreover, it can vary from object to object. 
As a first approximation, it seem sensible to set $\mathcal{X}=1$, i.e. to assume that stellar winds are smooth on average. In this case, measuring the easily observable $\mathrm{H} \alpha$ emission line can provide the mass-loss rate.

Puls et al. (1996) refined the theory of $\mathrm{H} \alpha$ formation, and presented scaling relations that connect $\dot{M}, v_{\infty}$, stellar parameters, and the $\mathrm{H} \alpha$ equivalent width. They applied this method to samples of O-stars in the Galaxy, the Large and the Small Magellanic Cloud. The results revealed a tight metallicity-dependent relation between the "radius modified stellar wind momentum rate", $\dot{M} v_{\infty} \sqrt{R_{*}}$, and the stellar luminosity. It was shown that $\mathrm{H} \alpha$ based mass-loss rates of $\mathrm{O}$ stars are in good general agreement with those derived from radio measurements (being free-free emission, the latter also depend on $\left\langle\rho^{2}\right\rangle$ processes). Moreover, the $\mathrm{H} \alpha$ based mass-loss rates of $\mathrm{O}$ stars were found in generally good agreement with theoretical predictions, i.e. in the range $10^{-4} \ldots 10^{-7} M_{\odot} \mathrm{yr}^{-1}$ depending on spectral type.

\section{Stellar wind clumping}

Despite of this large progress in measuring mass-loss rates, one has to be aware that the $\mathcal{X}=1$ assumption is not really justified, on the contrary there is strong evidence for stellar wind clumping (Hamann et al., 2008).

Clear evidence of wind inhomogeneity was provided by the detection of stochastic variability in the He II $\lambda 4686 \AA$ emission line in the spectrum of an $\mathrm{O}$ supergiant (Eversberg et al., 1998), explained by clump propagation. Line-profile variability of $\mathrm{H} \alpha$ was seen in a large sample of O-type supergiants, and attributed to the presence of shell fragments in structured winds (Markova et al., 2005). Prinja and Massa (2010) demonstrated that the winds of B supergiants are clumped by using spectral diagnostics. In a recent study, Martins et al. (2015) showed that spectral lines of OB supergiants are variable on various time scales likely because of the wind structuring. The line-profile variations in a sample of WR and O stars were monitored by Lépine and Moffat (1999, 2008). The observations were explained using a phenomenological model that depicts winds as being made up of a large number of randomly distributed, radially propagating clumps.

High-mass X-ray binaries (HMXBs) provide a confirmation of strong wind clumping. In some of these systems, a neutron star (NS) is in a close orbit deeply inside the stellar wind of an OB star. Accretion from the clumped stellar wind onto the NS powers strongly variable X-ray emission (e.g. Oskinova et al., 2012; Martínez-Núñez et al., 2014; Bozzo et al., 2016). van der Meer et al. (2005) studied the X-ray light curve and spectra of $4 \mathrm{U} 1700-37$ and concluded that the feeding of the NS by a strongly clumped stellar wind is consistent with the observed stochastic variability. Further evidence of donor wind clumping comes from the analysis of the X-ray spectra. Schulz et al. (2002) and Giménez-García et al. (2015) reviewed the spectroscopic results obtained with X-ray observatories for wind-fed HMXBs. They explained the observed spectra as originating in a clumped stellar wind, where cool dense clumps are embedded in rarefied X-ray pho- 
toionized gas. Fluorescence lines were used to trace wind clumps that are located close to the photosphere in a case of B-type supergiant donor (Torrejón et al., 2015).

The first X-ray spectra of putatively single O-type stars were obtained with the Einstein X-ray observatory. Stewart and Fabian (1981) used Einstein spectra of the O supergiant $\zeta$ Pup to determine its mass-loss rate. They applied a photoionization code and computed the stellar wind opacity using $\dot{M}$ as a model parameter. The X-ray based mass-loss rate was lower by a factor of a few than obtained from $\mathrm{H} \alpha$ and radio emission. As the most plausible explanation for this discrepancy it was suggested that the mass-loss rate measured from $\mathrm{H} \alpha$ and radio emission is overestimated because of wind clumping.

The Rosat X-ray spectrum of $\zeta$ Pup was investigated by Hillier et al. (1993). The wind was assumed to be smooth with a mass-loss rate $\dot{M}=5 \times 10^{-6} M_{\odot} \mathrm{yr}^{-1}$ consistent with the $\mathrm{H} \alpha$ measurement. It was found that the high opacity of the stellar wind should completely block soft X-rays $(<0.5 \mathrm{keV})$. However, since such soft X-rays are observed, it was concluded that a significant fraction of the X-ray emitting plasma is located far out in the wind, at distances $>100 R_{*}$.

The launch of XMM-Newton and Chandra X-ray telescopes made high-resolution Xray spectroscopy possible. The X-ray spectroscopic diagnostics allows to constrain the location of hot plasma emitting X-rays, and to probe the wind opacity (Macfarlane et al., 1991; Porquet et al., 2001). The analysis of X-ray spectra unambiguously showed that hot plasma is located not only far out in the wind, but also close to the stellar photosphere at distances $<1.5 R_{*}$, and that the wind opacity is much lower than expected from the $\mathrm{H} \alpha$ derived mass-loss rates (e.g. Waldron and Cassinelli, 2001; Kahn et al., 2001; Cassinelli et al., 2001).

Among others, the following two ways were suggested to explain the X-ray data: stellar wind clumping reduces the wind opacity and allows radiation to escape even for high mass-loss rates (Feldmeier et al., 2003; Oskinova et al., 2004), and stellar mass-loss rates are much lower than found from $\rho^{2}$ based diagnostics (Kramer et al., 2003).

\section{4. $\mathrm{P} \vee$ and the discordance of mass-loss rates diagnostics}

Massa et al. (2003) studied Far Ultraviolet Spectroscopic Explorer (FUSE) spectra of O stars in the Large Magellanic Cloud using the SEI method. They highlighted the importance of the $\mathrm{P}_{\mathrm{V}} \lambda \lambda 1117,1128 \AA$ resonance doublet for mass-loss diagnostics. This resonance doublet is never saturated because of the low phosphorus abundance (e.g. 1000 times less than carbon) making it specially suitable for mass-loss diagnostics. Moreover, $\mathrm{PV}$ is the dominant ionization stage in $\mathrm{O}$ stars, hence its ionization fraction is nearly unity (Krtička and Kubát, 2012). However, the analysis of observed spectra by Massa et al. (2003) revealed much weaker lines of $(\mathrm{PV})$ than expected if the $\mathrm{H} \alpha$ based massloss rates were true. It was concluded that the weak PV lines imply that either $\dot{M}$ is very low, or the assumed abundance of phosphorus is too large, or the winds are strongly 
clumped.

Bouret et al. (2005) and Fullerton et al. (2006) demonstrated the severe discordance of mass-loss rates empirically obtained from the $\rho^{2}$ - (such as $\mathrm{H} \alpha$ and radio) and the $\rho$ based (such as resonance lines, e.g. P v) diagnostic methods. The mass-loss rates measured from the UV lines were found to be at least an order of magnitude lower than those measures from $\mathrm{H} \alpha$. The UV line diagnostics were considered more reliable than $\mathrm{H} \alpha$, because they do not depend on clumping.

To explain this discordance it was suggested that the clumping is very strong and therefore mass-loss rates measured from $\mathrm{H} \alpha$ have to be reduced by orders of magnitude. Thus, based on the UV and X-ray diagnostics, the empirically estimated mass-loss from O-type stars were found to be significantly lower that predicted by the theory. This severe reduction of mass-loss rates was the basis for Smith and Owocki (2006) to propose a new evolutionary scenario according to which the stars with initial mass above $40 \ldots 50 M_{\odot}$ loose the bulk of their mass not via radiatively driven stellar winds but via explosive eruptions, and to discuss the possibility that Wolf-Rayet stars may not be the descendents of such massive stars.

\section{Microclumping}

Clearly, it is necessary to re-evaluate how clumping is accounted for in the spectral analysis and re-investigate the assumptions on which the mass-loss diagnostics are based. The usual and but stringent approximation is that all clumps in stellar wind are opti- cally thin. This approximation, called microclumping or filling factor approach, was implemented in non-LTE codes already back in 1990s (Hamann and Koesterke, 1998; Hillier and Miller, 1999).

If we assume that the density is uniform inside the clumps while the interclump medium is void, then $\mathcal{X}=D$, where $D$ is the density enhancement within clumps as compared to a smooth model with same mass-loss rate $\dot{M}$. In this case, the volume filling factor of the clumps is $f_{\mathrm{V}}=D^{-1}$. In the models, the rate equations have to be solved only for the clump medium, where the density is $D \rho$ instead of $\rho$ as in the smooth case.

Consequently, in the radiative transfer equation, the smooth-wind opacity and emissivity $\kappa(\rho)$ and $\eta(\rho)$ must be replaced for a clumped wind by

$$
\kappa_{\mathrm{f}}=f_{\mathrm{V}} \kappa_{\mathrm{C}}(D \rho) \quad \text { and } \quad \eta_{\mathrm{f}}=f_{\mathrm{V}} \eta_{\mathrm{C}}(D \rho)
$$

where $\kappa_{\mathrm{C}}$ and $\eta_{\mathrm{C}}$ are the non-LTE opacity and emissivity of the clump matter. The atomic transitions that contribute to the opacity and emissivity scale with different powers of the density. For processes linear in density, $f_{\mathrm{V}}$ and $D$ cancel. However, empirical mass-loss diagnostics are often based on processes that scale with the square of the density (recombination lines, free-free emission). When the wind is clumped, the emitted flux is enhanced by a factor of $D$ compared to a homogeneous model with the same mass-loss rate. Consequently, when a given (free-free radio or or recombination-powered line) emission is analyzed with a model that accounts for microclumping, the derived mass-loss rate will be 
lower by a factor of $\sqrt{D}$ than obtained with a smooth-wind model (see Eq.4).

In WR star spectra, fitting the observed electron scattering wings of strong emission lines can be used to determine the clumping factor $D$ and its radial dependence (Hillier, 1991; Hamann and Koesterke, 1998). For WR stars this method yields typical clumping factors $D$ between 4 and 10 .

Unfortunately, this method is not applicable for $\mathrm{O}$ stars, since their spectra do not show suitably strong emission lines. Hence, $D$ has to be constrained indirectly, e.g. as as a model parameter. Often, a radial dependence of clumping parameter is allowed in the models. Puls et al. (2006) estimated that the clumping factor $D$ is about four times larger in the line-forming region, compared to the radio-emitting region far away from the star. From their multiwavelength analysis of a B supergiant spectrum Puebla et al. (2016) estimated that $f_{\infty}$, i.e. the smallest filling factor approached at large wind velocities $\left(v(r) \rightarrow v_{\infty}\right)$, is 0.01, while Bouret et al. (2012) derived $f_{\infty}=0.03 \ldots 0.06$ for their sample of $\mathrm{O}$ stars. These results imply that the derived mass-loss rates are reduced by a factor $4 \ldots 10$ compared to the smooth wind analyses.

As an interesting consequence, microclumping reducing the size of the photoionized region in HMXBs. Even the largest clumps in stellar wind are likely optically thin at hard X-ray wavelengths emitted by an accreting NS. The strong X-ray radiation photoionizes the wind region surrounding the NS (Hatchett and McCray, 1977), and affects wind driving (e.g. Krtička et al., 2015). In clumped stellar winds the recombinations are favored compared to the ionizations. Hence, the size of the area photoionized by X-rays is smaller in realistic clumped winds compared to the smooth wind case (Oskinova et al., 2012).

\section{Macroclumping}

While the microclumping approximation is very convenient, this approach is too stringent. In reality stellar winds clumps could be optically thick at some frequencies. This situation has to be accounted for in modeling, e.g. using the macroclumping approach.

Accounting for optically thick clumping is required in a large variety of problems, such as continuum driven winds in luminous blue variables (LBV), X-ray emission from massive stars, or resonance line formation in stellar winds.

\subsection{Grey opacity}

Shaviv (1998) and Shaviv (2000) considered the gray opacity in a stellar atmosphere, where the photon mean free path does not exceed the scale of inhomogeneities. In such multi-phase porous atmosphere the radiation is able to escape easier while exerting a weaker average force. For a continuum driven wind, a star with the same luminosity would experience considerably smaller massloss compared to the case of homogeneous atmosphere. This may explain the observations of the LBV star $\eta$ Car (Shaviv, 2000). Owocki et al. (2004) introduced a "porosity-length" formalism to derive a simple scaling for the 
reduced effective opacity and used this to obtain an associated scaling for the continuumdriven mass-loss rate from stars that formally exceed the Eddington limit. Quataert et al. (2016) developed analytical and numerical models of the properties of super-Eddington stellar winds. Brown et al. (2004) pointed out that optically-thick clumping leads to the reduction of multiple scattering and, consequently, photon momentum delivery.

\subsection{Continuum opacity}

Stellar winds of OB stars are effective in absorbing X-rays via bound-free and K-shell photoionization processes. This high continuum opacity is strongly wavelength dependent (e.g. Verner and Yakovlev, 1995). In inner wind regions (e.g. at $0.5 v_{\infty}$ ) of a typical $\mathrm{O}$ supergiant, the mean free path of a photon at soft X-ray wavelength (e.g. at $19 \AA$ ) is only $\sim 10^{-2} R_{*}$. Clumps may have larger sizes and, hence, be optically thick.

The geometrical shape of clumps is not observationally constrained. There is only limited evidence for an intrinsic polarization of O star (McDavid, 2000; Harries et al., 2002), albeit this may be theoretically expected for stars with stronger winds and strong clumping (Brown et al., 2000; Li et al., 2000; Davies et al., 2007). Sophisticated but 1-D hydrodynamic models predict that wind clumps are flattened structures, with only small extend in radial direction (Owocki et al., 1988; Feldmeier et al., 1997). An initial attempts to include the multi-dimensional nature of radiation transport in hydrodynamical simulations (Dessart and Owocki, 2005) finds that damping of lateral velocity fluctuations isolates az- imuthal zones, leading to azimuthal incoherence down to the grid scale. This might indicate that the wind clumps might be rather somewhat elongated in radial direction (see also Gomez and Williams, 2003).

In either case, the continuum opacity of the wind consisting of such structures is anisotropic. Isotropic opacity is only adequate if the wind clumps are spherical.

The general solution of radiative transfer for the case of continuum non-gray opacity in an expanding inhomogeneous stellar wind was presented by Feldmeier et al. (2003). The optically thin and thick clumping as well as bridging cases were included in the theory. The formalism was developed in the context of X-ray lines emitted by an optically thin hot plasma that is attenuated in a fragmented cool stellar wind by strong continuum opacity. An analytic description for the effective isotropic and anisotropic opacity was found. It was pointed out that in case of isotropic opacity (e.g. spherical clumps) the line profiles are identical to those emerging from a smooth wind. On the other hand, in case of anisotropic opacity, the line profiles are characteristically different (Oskinova et al., 2006). The statistical solutions were verified by 2.5-D Monte-Carlo simulations (Oskinova et al., 2004) that allow nonconstant and non-monotonic distributions of mass-absorption coefficients and filling factors. Example model X-ray lines are shown in Fig. 1. As can be seen from this figure, the lines computed with clumped wind models are stronger compared to those computed with the smooth wind model for the same mass-loss rate. Also, the anisotropic wind 


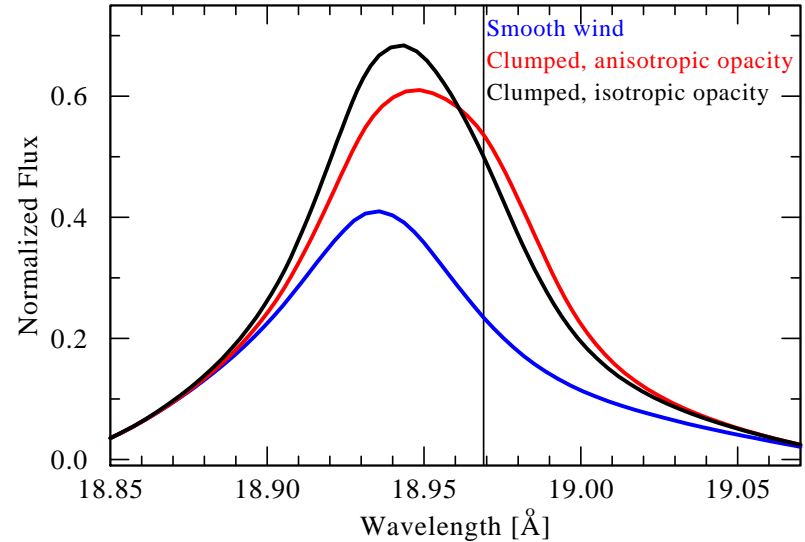

Figure 1: Three model line profiles of O VII in the X-ray spectrum of a O9II star. Except of clumping properties, all other model parameters are the same, $\log \dot{M}=-6.4\left[M_{\odot} \mathrm{yr}^{-1}\right], v_{\infty}=2000 \mathrm{~km} \mathrm{~s}^{-1}, \beta=0.8$. The onset of X-ray emission is at $1.2 R_{*}$. The blue line shows the line emerging from a smooth wind. The red line is a clumped wind model with $L=1.3$ $\left(\approx 2 \times 10^{4}\right.$ clumps between $1.2 R_{*}$ and $\left.100 R_{*}\right)$ and an anisotropic opacity (the clumps are flat in radial direction). The black line is for the same parameters, but now for isotropic opacity (e.g. the clumps have spherical shape). The vertical line denotes the rest frame line frequency. The model profiles are convolved with the instrumental profile of the HETGS MEG spectrograph on board of the Chandra X-ray telescope.

opacity leads to a more symmetric and less blues-shifted line profile.

Assume that the flow of clumps that constitutes the wind obeys the equation of continuity. The number of clumps per unit volume is $n(r) \equiv n_{0} v(R)^{-1} R^{-2}$, where $n_{0}\left[\mathrm{~s}^{-1}\right]$ is a constant. The effective opacity of the clumped wind, $\kappa_{\text {eff }}$, is the product of the clump number density $n(r)$, clump crosssection $\sigma_{\mathrm{C}}$, and the probability of an X-ray photon getting absorbed when it encounters a clump $\mathcal{P}=1-\exp \left(-\tau_{\mathrm{C}}(\nu)\right)$, where $\tau_{\mathrm{C}}(\nu)$ is the optical depth of the clump. The latter can be expressed as

$$
\tau_{\mathrm{C}}(\nu)=\kappa_{\nu} D \rho l R_{*},
$$

where $r=R / R_{*}$ and $l$ is the geometrical size of the clump expressed in $R_{*}$, and $\kappa_{\nu}$ $\left[\mathrm{cm}^{2} \mathrm{~g}^{-1}\right]$ is the mass absorption coefficient determining the continuum opacity (Feldmeier et al., 2003; Oskinova et al., 2011a). Due to the strong wavelength dependence of $\kappa_{\nu}$, a clump may be optically thick at longer wavelengths but thin at shorter ones. Evaluating the wind optical depth as an integral over effective opacity along the line-of-sight $z$ gives the optical depth:

$$
\tau_{\mathrm{w}}=n_{0} \int_{z_{\mathrm{em}}}^{\infty} \frac{\sigma_{\mathrm{C}}}{v(r) r^{2}}\left(1-\mathrm{e}^{-\tau_{\mathrm{C}}(\nu)}\right) \mathrm{d} z .
$$

Note that this integral starts from the $z$ coordinate of the X-ray emitter. The optical cross-section of spherical clumps (balls) is isotropic: $\sigma_{\mathrm{C}} \propto r^{2}$. For clumps in the form of shell-fragments (pancakes), the cross section depends on the projection angle as $\sigma_{\mathrm{C}} \propto|\mu| r^{2}$, where $\mu$ is the direction cosine. For the latter case, the integral Eq. 7 has a special property, because $\mathrm{d} z=\mathrm{d} r / \mu$. Hence, for X-ray line emitters in the front hemisphere which are located at some given radius, the $\mu$ dependence cancels out, i.e. all blue-shifted frequencies encounter the same optical depth and thus the same wind absorption. Only for the emitters in the back hemisphere, which create the red line wing, the optical depth increases with increasing redshift (Oskinova et al., 2004). 
In case when all clumps are optically thick, the emergent flux in the line is determined by the radiation leaking between these opaque clumps, and depends only on the clump shape and their geometrical distribution (Feldmeier et al., 2003; Oskinova et al., 2004). On the other hand, when all clumps are optically thin, the radiative transfer is not affected by the geometrical distribution of clumps and the line shape is the same as in case of smooth winds. It is important to remember, however, that the clump optical depth is a function of wavelength. Thus, it is most likely that neither of these two limiting cases (only optically thin or thick clumps) describe realistic winds.

Thus a more gray opacity and more symmetric lines are expected from clumped winds compared to smooth ones. This agrees with observations, namely nearly symmetric Xray line profiles, and the similarity of their profiles in the soft and hard parts of spectrum (Waldron and Cassinelli, 2001, 2007; Kramer et al., 2003). An alternative explanation could be provided by reduced mass-loss rates (Kramer et al., 2003; Leutenegger et al., 2013).

Owocki and Cohen (2006) and Leutenegger et al. (2013) used their porosity formalism to describe X-ray lines emerging from inhomogeneous stellar winds. They found that a substantial reduction in wind absorption requires quite large porosity lengths, and claimed that such large lengths $\left(\sim 1 R_{*}\right)$ are unphysical. The basis for these claims is the outcome from first 2-D models of nonstationary winds that were not capable to produce laterally coupled structures in the winds (Dessart and Owocki, 2005). However, these isothermal models are not capable to explain the X-ray emission from stellar winds either. Therefore it is not yet clear how qualitatively robust are their predictions on the size of the clumps. Moreover, the cool wind opacity has a strong wavelength dependence. Therefore, for harder radiation the clumps will remain optically thin even for very large porosity lengths, while for the softer radiation even small clumps will be optically thick. E.g., in the wind of the prototypical $\mathrm{O}$ star $\zeta$ Pup, clumps located at $2 R_{*}$ and with geometrical size of $0.07 R_{*}$ will be optically thick for the radiation in the Nex $\lambda 12.13$ line (Oskinova et al., 2011a).

Hervé et al. (2012) provided a careful comparison of the macroclumping and porosity formalisms and concluded that they are "essentially equivalent to first order". In a way this is not surprising, because in the porosity formalism the exact integrals (Feldmeier et al., 2003; Oskinova et al., 2006) are simplified using Taylor expansions. Hervé et al. (2012) modeled the X-ray spectra of O-stars. They concluded that including porosity does not improve the line fits. On the other hand, they used the radial dependence of X-ray filling factor as a model parameter. They found that such radial dependence is required to provide a suitable fit to the observed X-ray spectrum of an $\mathrm{O}$ supergiant. Leutenegger et al. (2013) incorporated the porosity formalism in a standard X-ray spectra fitting software. Fitting observed X-ray spectra, they found that neither porosity nor radial dependent filling factors improve the line fits, and concluded that these effects are not important and can be neglected. However, all 
recent studies of X-ray spectra of $\mathrm{O}$ stars by means of sophisticated non-LTE models show that simple smooth wind models with constant filling factors are incapable to explain the multiwavelength spectroscopic observations. Either macroclumping or radially dependent $\mathrm{X}$-ray filling factors have to be included in the models to reproduce the observations adequately (Hervé et al., 2012; Shenar et al., 2015; Rauw et al., 2015; Puebla et al., 2016).

Apart from the absorption of X-rays in clumped stellar winds, the problem of X-ray absorption in the interstellar medium also requires an adequate treatment of inhomogeneities. Wilms et al. (2000) provided a solution for this problem by including the selfshielding of the dust grains, and derived analytical expressions for the optical depth of the grains, assuming that the grains can be approximated as spheres.

\subsection{Line opacity}

Oskinova et al. (2007) realized that since the optical depth in the UV resonance lines is high and the line photon mean free path is short, the wind inhomogeneities are likely to be optically thick at these wavelengths. This is in agreement with Prinja and Massa (2010), who found spectroscopic evidence of optically thick clumps in the winds of B supergiants by measuring the ratios between the radial optical depths of the red and blue components of the SiIV doublets. From atomic physics this ratio should be exactly two. However, in all observed stars, only values $<2$ were found. This was interpreted as a direct signature of optically thick clumping.
To understand how optically thick clumping affects the radiative transfer in resonance lines, it is useful to consider the Sobolev approximation. According to this approximation, only matter close to the constant radial velocity surface contributes to the line optical depths. In a clumped wind, this surface will be porous (Fig. 2). Moreover, the opacity depends not only on the geometrical matter distribution, but also on the Sobolev length. For a smooth monotonic velocity, the Sobolev length is given by $v_{\mathrm{D}}(\mathrm{d} v / \mathrm{d} r)^{-1}$. In the original Sobolev approximation, $v_{\mathrm{D}}$ denotes the Doppler width by thermal broadening. Generalized to a clumpy medium, $v_{\mathrm{D}}$ can be considered as the dispersion of velocities inside an individual clump (Oskinova et al., 2007; Šurlan et al., 2012). Hence, the smaller the velocity dispersion within each clump, the narrower is the constant radial velocity surface. Consequently, a smaller number of clumps can contribute to the effective opacity, farther reducing it. In principle, the parameter $v_{\mathrm{D}}$ could be estimated from observations using spectroscopic techniques (e.g. Šurlan et al., 2013; Simón-Díaz and Herrero, 2014; Sander et al., 2015).

Owocki (2008) considered the special situation of a strongly non-monotonic wind velocity, motivated by dynamical simulations of the line-driven instability (Owocki et al., 1988; Feldmeier et al., 1997). At present, these dynamic models are limited to 1-D geometry, and it is not clear whether the same strong shocks would be seen in multidimensional models (Gomez and Williams, 2003; Dessart and Owocki, 2005). Moreover, observations of X-ray variability in massive 
stars, so far, do not confirm the 1-D model predictions on the temporal behavior of X-ray emission - no X-ray variability attributable to strong stochastic shocks is observed (e.g. Oskinova et al., 2001; Nazé et al., 2013). Owocki (2008) suggested that the strongly non-monotonic wind velocity seen in $1-\mathrm{D}$ models should lead to a reduction of the resonance line strength that is insensitive to spatial scales. This suggestion was in detail examined using their 2-D models by Sundqvist et al. (2010), who indeed found a reduction in the line strengths. However, the 3-D models (see Section 7) revealed that strongly nonmonotonic wind velocity has only moderate effect on the effective opacity. Moreover, it seems of limited importance to discuss the porosity and non-monotonic velocity effects separately. Both are playing role in establishing the line opacity and are consistently included in the macroclumping formalism .

To investigate the effects of macroclumping, an approximate treatment of the effective opacity was developed by Oskinova et al. (2007). A correction factor for macroclumping that can be easily included in a sophisticated non-LTE codes was derived. This treatment introduces a next level of complexity compared to microclumping, but is still quite approximate. Nevertheless, the validity of this approach is confirmed by 3-D Monte Carlo models (see Section 7).

In this macroclumping approximation, it is assumed that the clumps are statistically distributed, having an average separation $L$ between their centers $\left(L(r)\right.$ is expressed in $R_{*}$ and varies with radial location); hence, the volume filling factor is $f_{V}=l^{3} / L^{3}$, or

$$
D=L^{3} / l^{3} .
$$

From Eq. (8) it follows that

$$
n(r) \equiv L^{-3}=D^{-1} l^{-3},
$$

where $n_{\mathrm{C}}$ denotes the number density of the stochastically distributed clumps.

Optical depth across a clump can be approximated as $\tau_{\mathrm{C}}=\kappa_{\mathrm{C}} D \rho l R_{*}$. This is similar to the Eq. (6), except that now $\kappa_{\mathrm{C}}$ is the non-LTE opacity (see Eq.5). Recalling that $f_{\mathrm{V}}=D^{-1}$ yields

$$
\tau_{\mathrm{C}}=\kappa_{\mathrm{f}} D^{2 / 3} L .
$$

The effective opacity $\kappa_{\text {eff }}$ of the clumpy medium is obtained in analogy to the usual opacity from atomic absorbers:

$$
\kappa_{\mathrm{eff}}=n \sigma_{\mathrm{C}} .
$$

Here $\sigma_{\mathrm{C}}$ is the effective cross section of a clump. "Effective" means that the geometrical cross section, $l^{2}$, is multiplied by the fraction of photons that is absorbed when crossing the clump (Feldmeier et al., 2003),

$$
\sigma_{\mathrm{C}}=l^{2}\left(1-e^{-\tau_{\mathrm{C}}}\right) \text {. }
$$

Combining Eqs. (9), (11), and (12) yields

$$
\kappa_{\mathrm{eff}}=(D l)^{-1}\left(1-e^{-\tau_{\mathrm{C}}}\right) .
$$

Noticing that $(D l)^{-1}=\kappa_{\mathrm{f}} / \tau_{\mathrm{C}}$, the scaling of the effective opacity with the opacity obtained in the microclumping approximation can be written as:

$$
\kappa_{\mathrm{eff}}=\kappa_{\mathrm{f}} \frac{1-e^{-\tau_{\mathrm{C}}}}{\tau_{\mathrm{C}}} \equiv \kappa_{\mathrm{f}} C_{\text {macro }} .
$$




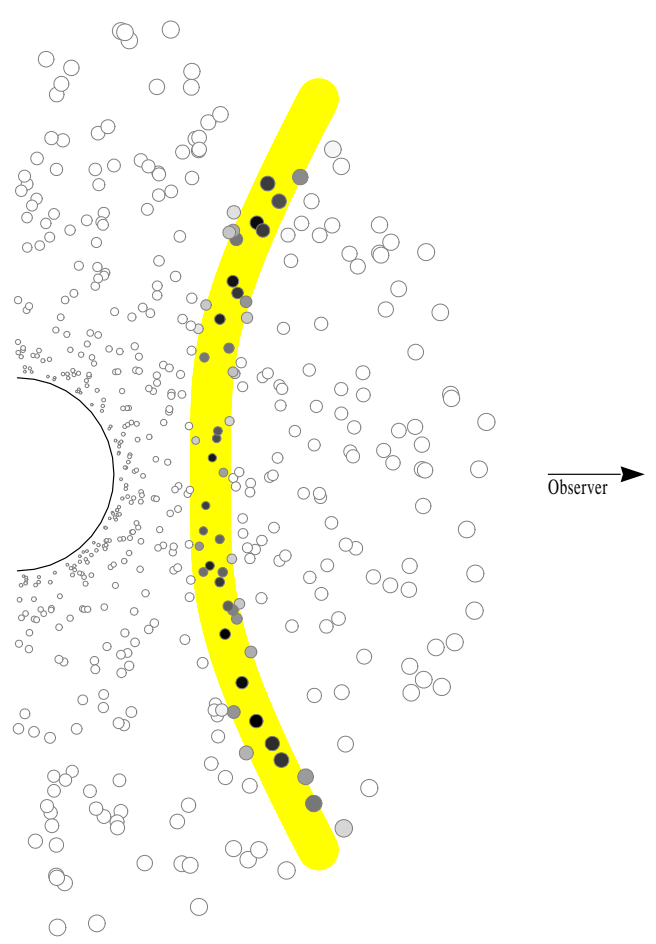

Figure 2: Sketch of a clumped stellar wind. In a smooth wind, rays of a given observer's frame frequency encounter line opacity only close to the "constant radial velocity surface" (thick shaded line). In a clumpy wind, assuming that the clumps move with the same velocity law as for the homogeneous wind, only those clumps interact with the ray that lie close to the corresponding constant radial velocity surface (dark-shaded circles). All other clumps are transparent (open circles) if the continuum opacity is small, so the wind is porous with respect to line transfer, even when the total volume is crowded with clumps. Adopted from Oskinova et al. (2007)

The factor $C_{\text {macro }}$ thus describes how macroclumping changes the opacity, compared to the microclumping limit. Note that for optically thin clumps $\left(\tau_{C} \ll 1\right)$ the microclumping approximation $\left(\kappa_{\text {eff }} \approx \kappa_{\mathrm{f}}\right)$ is recovered. For optically thick clumps $\left(\tau_{C} \gtrsim 1\right)$, however, the effective opacity is reduced by a factor $C_{\text {macro }}$. Since a detailed radiative transfer calculations inside each clump is yet impossible, the same reduction factor is applied to the corresponding emissivities, i.e. the non-LTE source is unchanged.

Synthetic spectra were computed using this macroclumping formalism and compared to the observed spectra of an O-type supergiant (Oskinova et al., 2007). It was shown that while the $\mathrm{H} \alpha$ line is not affected by macroclumping, the $\mathrm{P} V$ resonance doublet becomes significantly weaker and the massloss rate measured from it becomes higher when macroclumping is accounted for. Thus, it was demonstrated that including macro- 
clumping in the spectral analysis resolves the problem of discordant mass-loss rates obtained using $\rho$ - and $\rho^{2}$-diagnostics.

Importantly, Petrov et al. (2014) pointed out that for cooler B supergiants macroclumping should play a significant role in the formation of the $\mathrm{H} \alpha$ line, altering $\mathrm{H} \alpha$-based mass-loss rates.

To summarize, compared to microclumping which is mainly described by the parameter $D$ and its radial dependence, the macroclumping formalism requests at least one additional parameter $-L$. A sensible choice for this parameter could be made from careful consideration of the observed spectrum. Shenar et al. (2015) conducted a multiwavelength (X-ray to optical), non-LTE spectroscopic analysis of the bright eclipsing spectroscopic binary, $\delta$ Ori A (O9.5II). Motivated by hydrodynamic studies (Feldmeier et al., 1997; Runacres and Owocki, 2002), they assumed that the clumping initiates at $r=1.1 R_{*}$ and grows to its maximum contrast of $D=10$ at $r \sim 10 R_{*}$. A radius dependent macroclumping parameter $L$ was used, with $L=0.5 R_{*}$ in the low wind regions. The analysis confirmed the strong effect of macroclumping on the resonance lines. The $\mathrm{H} \alpha$ line, as well as the photospheric features, are hardly affected. The mass-loss rate derived from this multiwavelength spectral analysis was found to be in a good agreement with those theoretically predicted (Vink et al., 2001).

For comparison, a multi-wavelength (Xray to optical) analysis, based on the nonLTE models, of the B0 Ia-supergiant $\epsilon$ Ori did not include macroclumping. In order to describe the resonance line of Si IV, a micro- clumping parameter $f_{\infty}<0.01$ had to be adopted (Puebla et al., 2016). The corresponding mass-loss rate is at least one order of magnitude lower than prescribed by the Vink et al. (2001) recipe. (As an alternative Puebla et al. (2016) discuss possible problems with their model wind ionization structure.)

\section{Radiative transfer using realistic 3- D Monte-Carlo wind models}

The statistical treatment of macroclumping briefly outlined in section 6.3 provides a first approximation for radiative transfer in clumped winds. While this approximation could be easy included in large codes, full 3D models of clumped winds are required for in-depth studies. For the special case of line scattering, i.e. for resonance lines, such model was developed by Šurlan et al. (2012) and applied for the analysis of observed spectra by Surlan et al. (2013).

To study the basic effects of clumping on the resonance line formation (both singlets and doublets) in the 3-D Monte-Carlo code, a core-halo model was adopted. Only the line opacity is taken into account. Pure scattering and Doppler broadening are considered, and complete redistribution is assumed.

To solve the radiative transfer throughout the clumped wind, first a snapshot of the clump distribution is generated. Then, using the Monte Carlo approach the photons are followed along their paths. The density and velocity of the wind can be arbitrarily defined in a 3-D space. The calculations are carried out in the comoving frame following the prescriptions by Hamann (1980). 
The velocity field has three arbitrary Cartesian components. For simplicity, the velocity field is assumed radial and for the underlying smooth wind the standard $\beta$-velocity law is adopted. Non-monotonic velocities are also incorporated (Šurlan et al., 2012). The velocity inside the $i$-th clump is parametrized as

$$
v(r)=v_{\beta}\left(r_{\mathrm{i}}^{\mathrm{c}}\right)-m v_{\beta}(r) \frac{r-r_{\mathrm{i}}^{\mathrm{c}}}{l_{i}},
$$

where $l_{i}$ is the radius of the $i$-th clump, $r_{i}^{\mathrm{c}}$ is the absolute position of the center of the $i$-th clump, $v_{\beta}\left(r_{i}^{\mathrm{c}}\right)$ is the velocity determined according to $\beta$-velocity law at the position $r_{i}^{\mathrm{c}}$, and the velocity dispersion is $v_{\text {dis }}(r)=$ $m v_{\beta}(r)$, where $m(0<m \leq 1)$ is the velocity deviation parameter (free parameter of the model). Thus, Eq. (15) introduces a negative velocity gradient inside clumps, while the center of the clump moves according to a $\beta$-velocity law.

For the opacity, the parametrization of Hamann (1980) is used. Allowing for an arbitrary optical depth, clumps can be optically thick in the cores of resonance lines, while they remain optically thin at all other frequencies. For simplicity a spherical shape of the clumps is assumed, with a radius that varies with the distance $r$ from the star, $l=$ $l(r)$. The density inside clumps is assumed to be higher by the clumping factor $D$ than the smooth wind density at same $r$.

While for the statistical treatment we assumed that the interclump medium is void (Section 6), observations seems to indicate otherwise. The presence of a tenuous interclump medium is supported by the X- ray emission from O-type stars and by their strong lines of overionized ions, e.g. Ovi $\lambda \lambda 1032,1038 \AA$ (Cassinelli and Olson, 1979; Zsargó et al., 2008). The interclump medium is specified in the 3-D Monte Carlo models by the interclump density parameter $d$ $(0 \leq d<1)$, which is assumed to be depth independent. The interclump medium is rarefied by the factor $d$ compared to the smooth wind density. For the case of dense clumps and non-void interclump medium, the mass of the wind is distributed between clumps and interclump medium, and the clump filling factor becomes $f_{V}=(1-d) /(D-1)$, which may be depth dependent.

The detailed study by Surlan et al. (2012) showed that the different values for the parameters describing the clumping and the velocity field result in different strengths and shapes of the resonance lines (Fig. 3). The line profiles are sensitive to the spatial distribution of wind clumping. The density contrast, the clumping onset radius, and its radial distribution - all these play a role for the line formation.

Overall, the 3-D models confirm that macroclumping reduces the effective opacity in the resonance lines, and conclusively prove that in a realistic $3-\mathrm{D}$ wind with density and velocity variations the $\mathrm{P}$ Cygni profiles from resonance lines are different compared to smooth and stationary $3-\mathrm{D}$ winds. The parameter study showed that the key model parameter affecting the effective opacity is the clump separation, $L$.

Besides these general results, the 3-D models of radiative transfer in inhomogeneous stellar winds also brought new important in- 


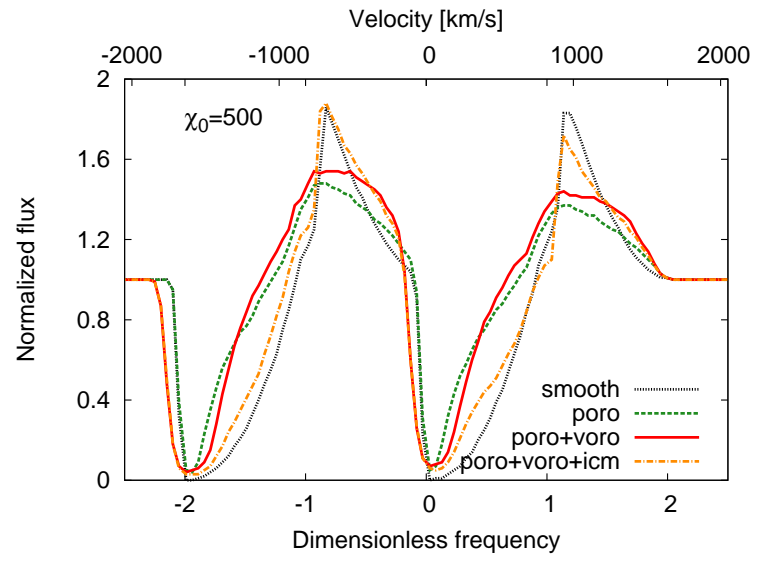

Figure 3: Effect of the macroclumping on a strong resonance doublet. The model accounts for a nonvoid interclump medium and for a velocity dispersion within the clumps. Four model lines are shown, computed with the same mass-loss rate. The black dashed line shows a smooth wind model, the green dashed lines illustrates the effect of macroclumping. The solid red line shows the same model as the green line, but now including velocity dispersion within clumps. The orange dash-dotted line shows the full model that includes also the effect of not-void interclump medium. Adopted from Fig. 10 in Šurlan et al. (2012)

sights that allow for a detail comparison with observations. E.g., the presence of an absorption dip near $v_{\infty}$ in a line profile was explained by the weakening of the macroclumping effects in outer regions of the stellar wind. A new diagnostic method for the onset of wind clumping was suggested, using the absorption dip at the line center. The study also confirmed the importance of the interclump medium, by demonstrating that a non-void interclump medium is required to reproduce the saturated spectral lines simultaneously with non-saturated ones. Finally, the study has highlighted that in any realistic wind,

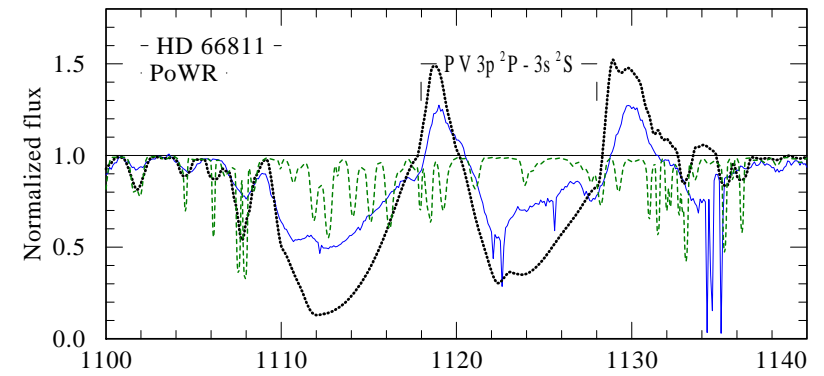

Figure 4: Comparison of observed and model spectra around $\mathrm{PV}$ resonance doublet in the O4I star HD 66811 ( $\zeta$ Pup). The thin solid-blue line is the observed spectrum. Dotted black is the PoWR model spectrum adopting $\dot{M}=2.5 \times 10^{-6} M_{\odot} \mathrm{yr}^{-1}$ and a depth dependent microclumping filling factor, which starts to deviate from the homogeneous wind $(D=$ 1) at about the sonic point $\left(5 \mathrm{~km} \mathrm{~s}^{-1}\right)$ and reaches $D=10$ at $v(r)=40 \mathrm{~km} \mathrm{~s}^{-1}$. The dashed-green lines are from the same model, but only accounting for the photospheric spectrum while the wind contribution is suppressed. Not accounting for macroclumping, the PoWR model predicts a much stronger $\mathrm{PV}$ doublet than observed.

non-monotonic velocity is always connected with density inhomogeneity. Therefore, their combined effects must be accounted for.

To compare the 3-D model with observations and measure mass-loss rates and other wind parameters, Šurlan et al. (2013) analyzed the optical and UV spectra of five O supergiant stars. This was done using a combination of the PoWR non-LTE stellar atmospheres and the Monte Carlo routine for the transfer of radiation in resonance lines.

As the first step, the PoWR models were fit to the observed optical spectra. From fitting the $\mathrm{H} \alpha$ line, mass-loss rate and the microclumping parameters were obtained and then fixed. The abundances, ionization stratification, and underlying photospheric spec- 
tra were also adopted from the PoWR models. Confirming previous studies, the UV resonance lines (especially $\mathrm{PV}$ ) were found to be too weak for the $\mathrm{H} \alpha$ based mass-loss rate (Fig. 4). Therefore, for these lines the 3-D Monte Carlo code was applied. The fixed, $\mathrm{H} \alpha$ based mass-loss rate was not changed during fitting, but the clumping parameters (density and velocity fields) could be adjusted (Fig. 5).

It turned out that the $\mathrm{H} \alpha$ and the $\mathrm{P}_{\mathrm{V}}$ lines can be fitted with the same mass-loss rate. Thus macroclumping resolves the discordance in mass-loss diagnostics as being due to the deficiencies in the treatment of clumping in standard modeling. The study also showed that the mass-loss rates obtained using macroclumping are only 1.5 to 2.5 times lower than predicted by the standard massloss recipe (Vink et al., 2001). The number of clumps required to obtain good fits, $\sim 10^{4}$ clumps up to $100 R_{*}$ compares well with the numbers observationally deduced from optical line variability studies and from X-ray observations of HMXBs (Eversberg et al., 1998; Fürst et al., 2010).

\section{What are the true empirical mass- loss rates of massive stars?}

The problem of realistic stellar mass-loss rates remains in the focus of massive star astrophysics. The concept of macroclumping, implemented so far as a statistical approach and as 3-D Monte-Carlo modeling for resonance lines, improved the empirical massloss diagnostics. Importantly, applications of these models revealed that the empirical mass-loss rates for O-stars that have been de-

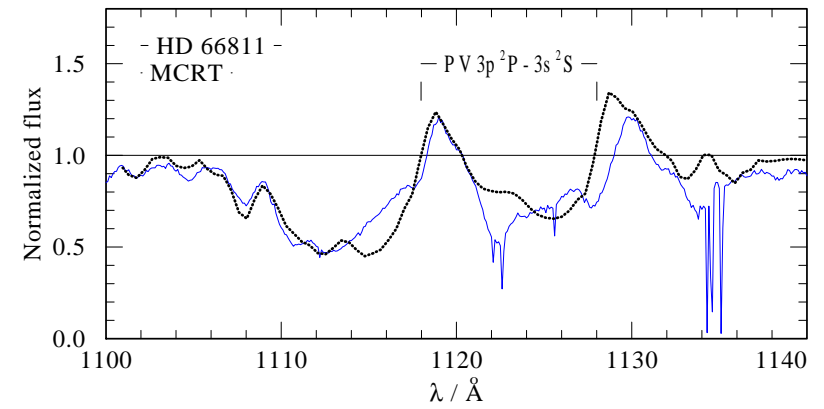

Figure 5: The same as in Fig. 4, but now the dotted black line is computed with the 3-D Monte Carlo wind model, using only the PoWR photospheric spectrum as input. The adopted mass-loss rate is again $\dot{M}=2.5 \times 10^{-6} M_{\odot} \mathrm{yr}^{-1}$. The line strength is significantly reduced compared to Fig. 4 despite the same adopted $\dot{M}$. See model details in Šurlan et al. (2013).

termined in the last years on the basis of the microclumping approach must not be drastically revised. The mass-loss rates of massive stars broadly agree with theoretical predictions (such as Vink et al., 2001). As a fortunate consequence, the mass-loss prescriptions used in the established evolutionary models for massive stars (e.g. Brott et al., 2011; Georgy et al., 2012) remain valid, at least roughly.

Since strong wind clumping modifies the effective opacity, it might also affect the radiative driving mechanism (e.g. Muijres et al., 2012; Sundqvist et al., 2014). The usual models that predict O-star mass loss rates (Vink et al., 2001) do not account for this. Due to the complexity of the problem and the likely connections between wind clumping and subphotospheric structures (Jiang et al., 2015), no self-consistent models of radiatively driven inhomogeneous stellar winds exist yet. The 
value of the mass-loss rate is determined by the physical conditions at the critical point at the base of the wind. Since the empirical mass-loss rates accounting for macroclumping, as reported above, are consistent within a factor of 1-3 with those predicted by the models from Vink et al. (2001), one may speculate that clumping is not yet strongly developed in these low-velocity layers.

Vink and Gräfener (2012) has shown that the mass-loss rates provided by current theoretical models for the Of/WNh-type stars are of the right order of magnitude. In evolved Wolf-Rayet stars the mass-loss rates are large and mainly diagnosed from their emissionline spectra (e.g. Hamann et al., 2006; Sander et al., 2012; Hainich et al., 2014). Similar to the O-stars, macroclumping may be required to achieve full accordance of mass-loss rate estimates using the UV resonance lines (Kubátová et al., 2015).

In this paper we did not cover the $\mathrm{B}$ and O-type stars of lower luminosities. The massloss rates estimated for these stars, based on IR, optical, and UV diagnostics, are much lower than expected (e.g. Martins et al., 2005; Marcolino et al., 2009; Najarro et al., 2011), posing the so-called "weak wind problem". Macroclumping alone cannot fully resolve this problem. A possible explanation is that a significant fraction of the wind in these stars is in a shock-heated phase and, therefore, can be detected only in X-rays (e.g. Cassinelli et al., 1994; Drew et al., 1994; Huenemoerder et al., 2012; Lucy, 2012).

Despite the success of the macroclumping model, one has to be aware of its limitations, such as a number of additional free param- eters, and the approximate treatment of the physical conditions within the clumps. Nevertheless, macroclumping is a step forward in our quest for realistic descriptions of stellar wind, which would not have been possible without the deep insights of V.V. Sobolev and his school on the physics of moving stellar envelopes.

The authors are indebted to an anonymous referee for the detailed and important comments and suggestions that significantly improved this paper. LO and BK acknowledge support from the DLR grant 50 OR 1508 and GA CR grant 16-01116S respectively.

\section{References}

Allen, C.W., 1973. Astrophysical quantities.

Bouret, J.C., Hillier, D.J., Lanz, T., Fullerton, A.W., 2012. Properties of Galactic early-type O-supergiants. A combined FUV-UV and optical analysis. A\&A 544, A67. doi:10.1051/0004-6361/201118594, arXiv: 1205.3075 .

Bouret, J.C., Lanz, T., Hillier, D.J., 2005. Lower mass loss rates in O-type stars: Spectral signatures of dense clumps in the wind of two Galactic O4 stars. A\&A 438, 301-316. doi:10.1051/0004-6361:20042531, arXiv:astro-ph/0412346.

Bozzo, E., Oskinova, L., Feldmeier, A., Falanga, M., 2016. Clumpy wind accretion in supergiant neutron star high mass X-ray binaries. A\&A 589, A102. 
doi:10.1051/0004-6361/201628341, arXiv: 1603.05187.

Brott, I., de Mink, S.E., Cantiello, M., Langer, N., de Koter, A., Evans, C.J., Hunter, I., Trundle, C., Vink, J.S., 2011. Rotating massive main-sequence stars. I. Grids of evolutionary models and isochrones. A\&A 530, A115. doi:10.1051/0004-6361/201016113, arXiv:1102.0530.

Brown, J.C., Cassinelli, J.P., Li, Q., Kholtygin, A.F., Ignace, R., 2004. Optically thick clumps - not the solution to the Wolf-Rayet wind momentum problem? A\&A 426, 323328. doi:10.1051/0004-6361:20040500, arXiv: astro-ph/0406685.

Brown, J.C., Ignace, R., Cassinelli, J.P., 2000. Hot star polarimetric variability and the nature of wind inhomogeneities. A\&A 356, 619-626.

Cassinelli, J.P., Cohen, D.H., Macfarlane, J.J., Sanders, W.T., Welsh, B.Y., 1994. X-ray emission from near-mainsequence B stars. ApJ 421, 705-717. doi:10.1086/173683.

Cassinelli, J.P., Miller, N.A., Waldron, W.L., MacFarlane, J.J., Cohen, D.H., 2001. Chandra Detection of Dopplershifted X-Ray Line Profiles from the Wind of $\zeta$ Puppis $(\mathrm{O} 4 \quad \mathrm{~F})$. ApJ 554, L55-L58. doi:10.1086/320916, arXiv: astro-ph/0104107.

Cassinelli, J.P., Olson, G.L., 1979. The effects of coronal regions on the X-ray flux and ionization conditions in the winds of OB supergiants and Of stars. ApJ 229, 304-317. doi:10.1086/156956.

Castor, J.I., 1970. Spectral line formation in Wolf-Rayet envelopes. MNRAS 149, 111127. doi:10.1093/mnras/149.2.111.

Castor, J.I., Abbott, D.C., Klein, R.I., 1975. Radiation-driven winds in Of stars. ApJ 195, 157-174. doi:10.1086/153315.

Castor, J.I., Lamers, H.J.G.L.M., 1979. An atlas of theoretical P Cygni profiles. ApJS 39, 481-511. doi:10.1086/190583.

Conti, P.S., Garmany, C.D., 1980. Mass loss from O-type stars. ApJ 238, 190-195. doi:10.1086/157972.

Davies, B., Vink, J.S., Oudmaijer, R.D., 2007. Modelling the clumpinginduced polarimetric variability of hot star winds. A\&A 469, 1045-1056. doi:10.1051/0004-6361:20077193, arXiv:0704.2569.

Dessart, L., Owocki, S.P., 2005. 2D simulations of the line-driven instability in hot-star winds. II. Approximations for the 2D radiation force. A\&A 437, 657666. doi:10.1051/0004-6361:20052778, arXiv: astro-ph/0503514.

Drew, J.E., Hoare, M.G., Denby, M., 1994. Soft X-ray observations of the early B giants Beta and Epsilon CMa. MNRAS 266, 917.

Evans, N.R., DeGioia-Eastwood, K., Gagné, M., Townsley, L., Broos, P., Wolk, S., 
Nazé, Y., Corcoran, M., Oskinova, L., Moffat, A.F.J., Wang, J., Walborn, N.R., 2011. The Search for Low-mass Companions of B Stars in the Carina Nebula Cluster Trumpler 16. ApJS 194, 13. doi:10.1088/0067-0049/194/1/13, arXiv:1103.1344.

Eversberg, T., Lépine, S., Moffat, A.F.J., 1998. Outmoving Clumps in the Wind of the Hot O Supergiant $\zeta$ Puppis. ApJ 494, 799-805. doi:10.1086/305218.

Feldmeier, A., Oskinova, L., Hamann, W.R., 2003. X-ray line emission from a fragmented stellar wind. A\&A 403, $217-$ 224. doi:10.1051/0004-6361:20030231, arXiv:astro-ph/0302516.

Feldmeier, A., Puls, J., Pauldrach, A.W.A., 1997. A possible origin for X-rays from O stars. A\&A 322, 878-895.

Fullerton, A.W., Massa, D.L., Prinja, R.K., 2006. The Discordance of Mass-Loss Estimates for Galactic O-Type Stars. ApJ 637, 1025-1039. doi:10.1086/498560, arXiv:astro-ph/0510252.

Fürst, F., Kreykenbohm, I., Pottschmidt, K., Wilms, J., Hanke, M., Rothschild, R.E., Kretschmar, P., Schulz, N.S., Huenemoerder, D.P., Klochkov, D., Staubert, R., 2010. X-ray variation statistics and wind clumping in Vela X-1. A\&A 519, A37. doi:10.1051/0004-6361/200913981, arXiv: 1005.5243.

Georgy, C., Ekström, S., Meynet, G., Massey, P., Levesque, E.M., Hirschi,
R., Eggenberger, P., Maeder, A., 2012. Grids of stellar models with rotation. II. WR populations and supernovae/GRB progenitors at $\mathrm{Z}=0.014$. A\&A 542, A29. doi:10.1051/0004-6361/201118340, arXiv: 1203.5243.

Giménez-García, A., Torrejón, J.M., Eikmann, W., Martínez-Núñez, S., Oskinova, L.M., Rodes-Roca, J.J., Bernabéu, G., 2015. An XMM-Newton view of FeK $\alpha$ in high-mass X-ray binaries. A\&A 576, A108. doi:10 . 1051/0004-6361/201425004.

Gomez, E.L., Williams, R.J.R., 2003. Nonspherical evolution of the line-driven wind instability. MNRAS 344, 725-740. doi:10.1046/j.1365-8711.2003.06864.x, arXiv:astro-ph/0305598.

Gräfener, G., Koesterke, L., Hamann, W.R., 2002. Line-blanketed model atmospheres for WR stars. A\&A 387, 244-257. doi:10.1051/0004-6361:20020269.

Grinin, V.P., 2001. Sobolev's Approximation. Astrophysics 44, 402-410. doi:10.1023/A : 1012884231706.

Groenewegen, M.A.T., Lamers, H.J.G.L.M., 1989. The winds of O-stars. I - an analysis of the UV line profiles with the SEI method. A\&AS 79, 359-383.

Hainich, R., Pasemann, D., Todt, H., Shenar, T., Sander, A., Hamann, W.R., 2015. Wolf-Rayet stars in the Small Magellanic Cloud. I. Analysis of the single WN stars. A\&A 581, A21. 
doi:10.1051/0004-6361/201526241, arXiv: 1507.04000.

Hainich, R., Rühling, U., Todt, H., Oskinova, L.M., Liermann, A., Gräfener, G., Foellmi, C., Schnurr, O., Hamann, W.R., 2014. The Wolf-Rayet stars in the Large Magellanic Cloud. A comprehensive analysis of the WN class. A\&A 565, A27. doi:10.1051/0004-6361/201322696, arXiv: 1401.5474.

Hamann, W.R., 1980. The expanding envelope of Zeta Puppis - A detailed UV-line fit. A\&A 84, 342-349.

Hamann, W.R., 1981. The expanding envelope of Tau Scorpii - A detailed UV-line fit. A\&A 100, 169-174.

Hamann, W.R., Gräfener, G., 2003. A temperature correction method for expanding atmospheres. A\&A 410, 993-1000. doi:10.1051/0004-6361:20031308.

Hamann, W.R., Gräfener, G., Liermann, A., 2006. The Galactic WN stars. Spectral analyses with lineblanketed model atmospheres versus stellar evolution models with and without rotation. A\&A 457, 1015-1031. doi:10.1051/0004-6361:20065052, arXiv: astro-ph/0608078.

Hamann, W.R., Koesterke, L., 1998. Spectrum formation in clumped stellar winds: consequences for the analyses of WolfRayet spectra. A\&A 335, 1003-1008.
Hamann, W.R., Oskinova, L.M., Feldmeier, A., 2008. Spectrum formation in clumpy stellar winds, in: Hamann, W.R., Feldmeier, A., Oskinova, L.M. (Eds.), Clumping in Hot-Star Winds, p. 75.

Harries, T.J., Howarth, I.D., Evans, C.J., 2002. Spectropolarimetry of O supergiants. MNRAS 337, 341-355. doi:10.1046/j.1365-8711.2002.05926.x.

Hatchett, S., McCray, R., 1977. X-ray sources in stellar winds. ApJ 211, 552-561. doi:10. 1086/154962.

Heger, A., Fryer, C.L., Woosley, S.E., Langer, N., Hartmann, D.H., 2003. How Massive Single Stars End Their Life. ApJ 591, 288-300. doi:10.1086/375341, arXiv: astro-ph/0212469.

Hervé, A., Rauw, G., Nazé, Y., Foster, A., 2012. Global Modeling of X-Ray Spectra Produced in O-type Star Winds. ApJ 748, 89. doi:10.1088/0004-637X/748/2/89, arXiv: 1201.4716 .

Hillier, D.J., 1991. The effects of electron scattering and wind clumping for early emission line stars. A\&A 247, 455-468.

Hillier, D.J., Kudritzki, R.P., Pauldrach, A.W., Baade, D., Cassinelli, J.P., Puls, J., Schmitt, J.H.M.M., 1993. The 0.1-2.5KEV X-Ray Spectrum of the O4F-STAR Zeta-Puppis. A\&A 276, 117.

Hillier, D.J., Miller, D.L., 1999. Constraints on the Evolution of Massive Stars through Spectral Analysis. I. The WC5 
Star HD 165763. ApJ 519, 354-371. doi:10.1086/307339.

Huenemoerder, D.P., Oskinova, L.M., Ignace, R., Waldron, W.L., Todt, H., Hamaguchi, K., Kitamoto, S., 2012. On the Weak-wind Problem in Massive Stars: X-Ray Spectra Reveal a Massive Hot Wind in $\mu$ Columbae. ApJ 756, L34. doi:10.1088/2041-8205/756/2/L34, arXiv: 1208.0820.

Jeffery, C.S., Hamann, W.R., 2010. Stellar winds and mass loss from extreme helium stars. MNRAS 404, 1698-1710. doi:10.1111/j.1365-2966.2010.16410.x, arXiv: 1001.4399.

Jiang, Y.F., Cantiello, M., Bildsten, L., Quataert, E., Blaes, O., 2015. Local Radiation Hydrodynamic Simulations of Massive Star Envelopes at the Iron Opacity Peak. ApJ 813, 74. doi:10.1088/0004-637X/813/1/74, arXiv: 1509.05417.

Kahn, S.M., Leutenegger, M.A., Cottam, J., Rauw, G., Vreux, J.M., den Boggende, A.J.F., Mewe, R., Güdel, M., 2001. High resolution X-ray spectroscopy of zeta Puppis with the XMM-Newton reflection grating spectrometer. A\&A 365, L312L317. doi:10.1051/0004-6361:20000093, arXiv:astro-ph/0011026.

Kramer, R.H., Cohen, D.H., Owocki, S.P., 2003. X-Ray Emission-Line Profile Modeling of O Stars: Fitting a Spherically Symmetric Analytic Wind-Shock Model to the Chandra Spectrum of $\zeta$ Puppis. ApJ 592, 532-538. doi:10.1086/375390, arXiv: astro-ph/0211550.

Krtička, J., Kubát, J., 2012. Influence of extreme ultraviolet radiation on the $\mathrm{P} V$ ionization fraction in hot star winds. MNRAS 427, 84-90. doi:10.1111/j.1365-2966.2012.21895.x, arXiv:1208.1834.

Krtička, J., Kubát, J., Krtičková, I., 2015. Xray irradiation of the winds in binaries with massive components. A\&A 579, A111. doi:10.1051/0004-6361/201525637, arXiv: 1505.03411.

Kubátová, B., Hamann, W.R., Todt, H., Sander, A., Steinke, M., Hainich, R., Shenar, T., 2015. Macroclumping in WR 136, in: Hamann, W.R., Sander, A., Todt, H. (Eds.), Wolf-Rayet Stars: Proceedings of an International Workshop held in Potsdam, Germany, 1-5 June 2015. Edited by Wolf-Rainer Hamann, Andreas Sander, Helge Todt. Universitätsverlag Potsdam, 2015., p.125-128, pp. 125-128.

Lamers, H.J.G.L.M., Cassinelli, J.P., 1999. Introduction to Stellar Winds.

Lamers, H.J.G.L.M., Cerruti-Sola, M., Perinotto, M., 1987. The 'SEI' method for accurate and efficient calculations of line profiles in spherically symmetric stellar winds. ApJ 314, 726-738. doi:10.1086/165100.

Lamers, H.J.G.L.M., Haser, S., de Koter, A., Leitherer, C., 1999. The Ionization in the 
Winds of O Stars and the Determination of Mass-Loss Rates from Ultraviolet Lines. ApJ 516, 872-886. doi:10.1086/307127.

Lépine, S., Moffat, A.F.J., 1999. Wind Inhomogeneities in Wolf-Rayet Stars. II. Investigation of Emission-Line Profile Variations. ApJ 514, 909-931. doi:10.1086/306958.

Lépine, S., Moffat, A.F.J., 2008. Direct Spectroscopic Observations of Clumping in O-Star Winds. AJ 136, 548 553. doi:10.1088/0004-6256/136/2/548, arXiv:0805.1864.

Leutenegger, M.A., Cohen, D.H., Sundqvist, J.O., Owocki, S.P., 2013. Constraints on Porosity and Mass Loss in O-star Winds from the Modeling of X-Ray Emission Line Profile Shapes. ApJ 770, 80. doi:10.1088/0004-637X/770/1/80, arXiv:1305.5595.

Li, Q., Brown, J.C., Ignace, R., Cassinelli, J.P., Oskinova, L.M., 2000. Wolf-Rayet wind structure and optical variability. A\&A 357, 233-240.

Lucy, L.B., 1971. The Formation of Resonance Lines in Extended and Expanding Atmospheres. ApJ 163, 95. doi:10.1086/150748.

Lucy, L.B., 1982. The formation of resonance lines in locally nonmonotonic winds. ApJ 255, 278-285. doi:10.1086/159826.

Lucy, L.B., 1983. The formation of resonance lines in locally nonmonotonic winds. II - an amplitude diagnostic. ApJ 274, 372-379. doi:10.1086/161453.

Lucy, L.B., 2012. Coronal winds powered by radiative driving. A\&A 544, A120. doi:10.1051/0004-6361/201118753, arXiv: 1201.0483.

Macfarlane, J.J., Cassinelli, J.P., Welsh, B.Y., Vedder, P.W., Vallerga, J.V., Waldron, W.L., 1991. Predicted extremeultraviolet line emission for nearby mainsequence B stars. ApJ 380, 564-574. doi:10.1086/170614.

Marcolino, W.L.F., Bouret, J.C., Martins, F., Hillier, D.J., Lanz, T., Escolano, C., 2009. Analysis of Galactic latetype $\mathrm{O}$ dwarfs: more constraints on the weak wind problem. A\&A 498, 837852. doi:10.1051/0004-6361/200811289, arXiv:0902.1833.

Markova, N., Puls, J., Scuderi, S., Markov, H., 2005. Bright OB stars in the Galaxy. II. Wind variability in $\mathrm{O}$ supergiants as traced by $\mathrm{H} \alpha$. A\&A 440, 1133 1151. doi:10.1051/0004-6361:20041774, arXiv:astro-ph/0505613.

Martínez-Núñez, S., Torrejón, J.M., Kühnel, M., Kretschmar, P., Stuhlinger, M., RodesRoca, J.J., Fürst, F., Kreykenbohm, I., Martin-Carrillo, A., Pollock, A.M.T., Wilms, J., 2014. The accretion environment in Vela X-1 during a flaring period using XMM-Newton. A\&A 563, A70. doi:10.1051/0004-6361/201322404, arXiv: 1401.2848. 
Martins, F., Marcolino, W., Hillier, D.J., Donati, J.F., Bouret, J.C., 2015. Radial dependence of line profile variability in seven O9-B0.5 stars. A\&A 574, A142. doi:10.1051/0004-6361/201423882, arXiv:1409.5057.

Martins, F., Schaerer, D., Hillier, D.J., Meynadier, F., Heydari-Malayeri, M., Walborn, N.R., 2005. On stars with weak winds: the Galactic case. A\&A 441, 735762. doi:10.1051/0004-6361:20052927, arXiv: astro-ph/0507278.

Massa, D., Fullerton, A.W., Sonneborn, G., Hutchings, J.B., 2003. Constraints on the Ionization Balance of Hot-Star Winds from FUSE Observations of $\mathrm{O}$ Stars in the Large Magellanic Cloud. ApJ 586, 996-1018. doi:10.1086/367786, arXiv: astro-ph/0211518.

McDavid, D., 2000. A Search for Intrinsic Polarization in O Stars with Variable Winds. AJ 119, 352-364. doi:10.1086/301186, arXiv: astro-ph/9909456.

Mihalas, D., Kunasz, P.B., Hummer, D.G., 1975. Solution of the comoving frame equation of transfer in spherically symmetric flows. I - Computational method for equivalent-two-levelatom source functions. ApJ 202, 465-489. doi:10.1086/153996.

Muijres, L.E., Vink, J.S., de Koter, A., Müller, P.E., Langer, N., 2012. Predictions for mass-loss rates and terminal wind velocities of massive O-type stars. A\&A 537,
A37. doi:10.1051/0004-6361/201015818, arXiv:1112.0944.

Najarro, F., Hanson, M.M., Puls, J., 2011. L-band spectroscopy of Galactic OB-stars. A\&A 535, A32. doi:10.1051/0004-6361/201016003, arXiv:1108.5752.

Nazé, Y., Oskinova, L.M., Gosset, E., 2013. A Detailed X-Ray Investigation of $\zeta$ Puppis. II. The Variability on Short and Long Timescales. ApJ 763, 143. doi:10.1088/0004-637X/763/2/143, arXiv: 1212.1554 .

Oskinova, L., Hamann, W.R., Ignace, R., Feldmeier, A., 2011a. X-rays, clumping and wind structures. Bulletin de la Societe Royale des Sciences de Liege 80, 54-66. arXiv: 1012.1857.

Oskinova, L.M., Clarke, D., Pollock, A.M.T., 2001. Rotationally modulated $\mathrm{X}$-ray emission from the single $\mathrm{O}$ star $\zeta$ Ophiuchi. A\&A 378, L21-L24. doi:10.1051/0004-6361:20011222.

Oskinova, L.M., Feldmeier, A., Hamann, W.R., 2004. X-ray emission lines from inhomogeneous stellar winds. A\&A 422, 675691. doi:10.1051/0004-6361:20047187, arXiv: astro-ph/0403707.

Oskinova, L.M., Feldmeier, A., Hamann, W.R., 2006. High-resolution Xray spectroscopy of bright O-type stars. MNRAS 372, 313-326. doi:10.1111/j.1365-2966.2006.10858.x, arXiv:astro-ph/0603286. 
Oskinova, L.M., Gayley, K.G., Hamann, W.R., Huenemoerder, D.P., Ignace, R., Pollock, A.M.T., 2012. High-resolution XRay Spectroscopy Reveals the Special Nature of Wolf-Rayet Star Winds. ApJ 747, L25. doi:10.1088/2041-8205/747/2/L25, arXiv:1202.1525.

Oskinova, L.M., Hamann, W.R., Feldmeier, A., 2007. Neglecting the porosity of hot-star winds can lead to underestimating mass-loss rates. A\&A 476, 13311340. doi:10.1051/0004-6361:20066377, arXiv:0704.2390.

Oskinova, L.M., Todt, H., Ignace, R., Brown, J.C., Cassinelli, J.P., Hamann, W.R., 2011b. Early magnetic Btype stars: X-ray emission and wind properties. MNRAS 416, 1456-1474. doi:10.1111/j.1365-2966.2011.19143.x, arXiv: 1106.0508.

Owocki, S.P., 2008. Dynamical simulation of the "velocity-porosity" reduction in observed strength of stellar wind lines, in: Hamann, W.R., Feldmeier, A., Oskinova, L.M. (Eds.), Clumping in Hot-Star Winds, p. 121.

Owocki, S.P., Castor, J.I., Rybicki, G.B., 1988. Time-dependent models of radiatively driven stellar winds. I - Nonlinear evolution of instabilities for a pure absorption model. ApJ 335, 914-930. doi:10.1086/166977.

Owocki, S.P., Cohen, D.H., 2006. The Effect of Porosity on X-Ray EmissionLine Profiles from Hot-Star Winds. ApJ
648, 565-571. doi:10.1086/505698, arXiv: astro-ph/0602054.

Owocki, S.P., Gayley, K.G., Shaviv, N.J., 2004. A Porosity-Length Formalism for Photon-Tiring-limited Mass Loss from Stars above the Eddington Limit. ApJ 616, 525-541. doi:10.1086/424910, arXiv: astro-ph/0409573.

Pauldrach, A., Puls, J., Kudritzki, R.P., 1986. Radiation-driven winds of hot luminous stars - Improvements of the theory and first results. A\&A 164, 86-100.

Petrov, B., Vink, J.S., Gräfener, G., 2014. On the $\mathrm{H} \alpha$ behaviour of blue supergiants: rise and fall over the bi-stability jump. A\&A 565, A62. doi:10.1051/0004-6361/201322754, arXiv:1403.4097.

Porquet, D., Mewe, R., Dubau, J., Raassen, A.J.J., Kaastra, J.S., 2001. Line ratios for helium-like ions: Applications to collisiondominated plasmas. A\&A 376, 11131122. doi:10.1051/0004-6361:20010959, arXiv: astro-ph/0107329.

Prinja, R.K., Massa, D.L., 2010. Signature of wide-spread clumping in $\mathrm{B}$ supergiant winds. A\&A 521, L55. doi:10.1051/0004-6361/201015252, arXiv:1007.2744.

Puebla, R.E., Hillier, D.J., Zsargó, J., Cohen, D.H., Leutenegger, M.A., 2016. $\mathrm{X}$-ray, UV and optical analysis of supergiants: $\epsilon$ Ori. MNRAS 456, 2907- 
2936. doi:10.1093/mnras/stv2783, arXiv: 1511.09365.

Puls, J., Kudritzki, R.P., Herrero, A., Pauldrach, A.W.A., Haser, S.M., Lennon, D.J., Gabler, R., Voels, S.A., Vilchez, J.M., Wachter, S., Feldmeier, A., 1996. O-star mass-loss and wind momentum rates in the Galaxy and the Magellanic Clouds Observations and theoretical predictions. A\&A 305, 171.

Puls, J., Markova, N., Scuderi, S., Stanghellini, C., Taranova, O.G., Burnley, A.W., Howarth, I.D., 2006. Bright OB stars in the Galaxy. III. Constraints on the radial stratification of the clumping factor in hot star winds from a combined $\mathrm{H}, \mathrm{IR}$ and radio analysis. A\&A 454, 625651. doi:10.1051/0004-6361:20065073, arXiv: astro-ph/0604372.

Puls, J., Vink, J.S., Najarro, F., 2008. Mass loss from hot massive stars. A\&A Rev. 16, 209-325. doi:10.1007/s00159-008-0015-8, arXiv:0811.0487.

Quataert, E., Fernández, R., Kasen, D., Klion, H., Paxton, B., 2016. SuperEddington stellar winds driven by nearsurface energy deposition. MNRAS 458, 1214-1233. doi:10.1093/mnras/stw365, arXiv: 1509.06370.

Rauw, G., Hervé, A., Nazé, Y., GonzálezPérez, J.N., Hempelmann, A., Mittag, M., Schmitt, J.H.M.M., Schröder,
K.P., Gosset, E., Eenens, P., UuhSonda, J.M., 2015. Simultaneous Xray and optical spectroscopy of the Oef supergiant $\lambda$ Cephei. A\&A 580, A59. doi:10.1051/0004-6361/201526057, arXiv: 1505.07714.

Runacres, M.C., Owocki, S.P., 2002. The outer evolution of instabilitygenerated structure in radiatively driven stellar winds. A\&A 381, 1015-1025. doi:10.1051/0004-6361:20011526.

Sander, A., Hamann, W.R., Todt, H., 2012. The Galactic WC stars. Stellar parameters from spectral analyses indicate a new evolutionary sequence. A\&A 540, A144. doi:10.1051/0004-6361/201117830, arXiv:1201.6354.

Sander, A., Shenar, T., Hainich, R., Gímenez-García, A., Todt, H., Hamann, W.R., 2015. On the consistent treatment of the quasi-hydrostatic layers in hot star atmospheres. A\&A 577, A13. doi:10.1051/0004-6361/201425356, arXiv: 1503. 01338.

Schulz, N.S., Canizares, C.R., Lee, J.C., Sako, M., 2002. The Ionized Stellar Wind in Vela X-1 during Eclipse. ApJ 564, L21-L25. doi:10.1086/338862, arXiv: astro-ph/0111489.

Shaviv, N.J., 1998. The Eddington Luminosity Limit for Multiphased Media. ApJ 494, L193-L197. doi:10.1086/311182.

Shaviv, N.J., 2000. The Porous Atmosphere of $\eta$ Carinae. ApJ 532, 
L137-L140. doi:10.1086/312585, arXiv:astro-ph/0002212.

Shenar, T., Oskinova, L., Hamann, W.R., Corcoran, M.F., Moffat, A.F.J., Pablo, H., Richardson, N.D., Waldron, W.L., Huenemoerder, D.P., Maíz Apellániz, J., Nichols, J.S., Todt, H., Nazé, Y., Hoffman, J.L., Pollock, A.M.T., Negueruela, I., 2015. A Coordinated X-Ray and Optical Campaign of the Nearest Massive Eclipsing Binary, $\delta$ Orionis Aa. IV. A Multiwavelength, Non-LTE Spectroscopic Analysis. ApJ 809, 135. doi:10.1088/0004-637X/809/2/135, arXiv: 1503.03476.

Simón-Díaz, S., Herrero, A., 2014. The IACOB project. I. Rotational velocities in northern Galactic O- and early B-type stars revisited. The impact of other sources of line-broadening. A\&A 562, A135. doi:10.1051/0004-6361/201322758, arXiv: 1311.3360 .

Smith, N., Owocki, S.P., 2006. On the Role of Continuum-driven Eruptions in the Evolution of Very Massive Stars and Population III Stars. ApJ 645, L45-L48. doi:10.1086/506523, arXiv:astro-ph/0606174.

Sobolev, V.V., 1947. Moving envelopes of stars. Leningrad: Leningrad State University Press; (in Russian). English edition: Cambridge, MA: Harvard University Press, 1960 .

Stewart, G.C., Fabian, A.C., 1981. The influence of mass loss on the observed X-ray spectra of early-type stars. MNRAS 197, 713-720.

Sundqvist, J.O., Puls, J., Feldmeier, A., 2010. Mass loss from inhomogeneous hot star winds. I. Resonance line formation in 2D models. A\&A 510, A11. doi:10.1051/0004-6361/200912842, arXiv:0911.3361.

Sundqvist, J.O., Puls, J., Owocki, S.P., 2014. Mass loss from inhomogeneous hot star winds. III. An effective-opacity formalism for line radiative transfer in accelerating, clumped two-component media, and first results on theory and diagnostics. A\&A 568, A59. doi:10.1051/0004-6361/201423570, arXiv: 1405.7800 .

Todt, H., Peña, M., Hamann, W.R., Gräfener, G., 2010. The central star of the planetary nebula $\mathrm{PB}$ 8: a Wolf-Rayet-type wind of an unusual WN/WC chemical composition. A\&A 515, A83. doi:10.1051/0004-6361/200912183, arXiv:1003.3419.

Torrejón, J.M., Schulz, N.S., Nowak, M.A., Oskinova, L., Rodes-Roca, J.J., Shenar, T., Wilms, J., 2015. On the Radial Onset of Clumping in the Wind of the B0I Massive Star QV Nor. ApJ 810, 102. doi:10.1088/0004-637X/810/2/102.

Šurlan, B., Hamann, W.R., Aret, A., Kubát, J., Oskinova, L.M., Torres, A.F., 2013. Macroclumping as solution of the discrepancy between $\mathrm{H} \alpha$ and $\mathrm{P}$ v mass loss diagnostics for O-type stars. A\&A 559, A130. 
doi:10.1051/0004-6361/201322390, arXiv: 1310.0449.

Šurlan, B., Hamann, W.R., Kubát, J., Oskinova, L.M., Feldmeier, A., 2012. Threedimensional radiative transfer in clumped hot star winds. I. Influence of clumping on the resonance line formation. A\&A 541, A37. doi:10.1051/0004-6361/201118590, arXiv:1202.4787.

van der Meer, A., Kaper, L., di Salvo, T., Méndez, M., van der Klis, M., Barr, P., Trams, N.R., 2005. XMM-Newton X-ray spectroscopy of the high-mass X-ray binary $4 \mathrm{U} 1700-37$ at low flux. A\&A 432, 9991012. doi:10.1051/0004-6361:20041288, arXiv: astro-ph/0412021.

Verner, D.A., Yakovlev, D.G., 1995. Analytic FITS for partial photoionization cross sections. A\&AS 109, 125-133.

Vink, J.S., de Koter, A., Lamers, H.J.G.L.M., 2001. Mass-loss predictions for $\mathrm{O}$ and $\mathrm{B}$ stars as a function of metallicity. A\&A 369, 574-588. doi:10.1051/0004-6361:20010127, arXiv: astro-ph/0101509.

Vink, J.S., Gräfener, G., 2012. The Transition Mass-loss Rate: Calibrating the Role of Line-driven Winds in Massive Star Evolution. ApJ 751, L34. doi:10.1088/2041-8205/751/2/L34, arXiv: 1205.0394.

Waldron, W.L., Cassinelli, J.P., 2001. Chandra Discovers a Very High Density XRay Plasma on the O Star $\zeta$ Orionis.
ApJ 548, L45-L48. doi:10.1086/318926, arXiv: astro-ph/0012190.

Waldron, W.L., Cassinelli, J.P., 2007. An Extensive Collection of Stellar Wind XRay Source Region Emission Line Parameters, Temperatures, Velocities, and Their Radial Distributions as Obtained from Chandra Observations of 17 OB Stars. ApJ 668, 456-480. doi:10.1086/520919, arXiv:0707.0024.

Wilms, J., Allen, A., McCray, R., 2000. On the Absorption of X-Rays in the Interstellar Medium. ApJ 542, 914-924. doi:10.1086/317016, arXiv: astro-ph/0008425.

Zsargó, J., Hillier, D.J., Bouret, J.C., Lanz, T., Leutenegger, M.A., Cohen, D.H., 2008. On the Importance of the Interclump Medium for Superionization: O VI Formation in the Wind of $\zeta$ Puppis. ApJ 685, L149-L152. doi:10.1086/592568, arXiv:0808.2233. 